\title{
On the behavior of the solutions of the system of rational difference equations

$$
x_{n+1}=\frac{x_{n-1}}{y_{n} x_{n-1}-1}, y_{n+1}=\frac{y_{n-1}}{x_{n} y_{n-1}-1}, z_{n+1}=\frac{x_{n} z_{n-1}}{y_{n}}
$$

\section{Abdullah Selcuk Kurbanli ${ }^{1 \star}$, Ibrahim Yalcinkaya ${ }^{1}$ and Ali Gelisken ${ }^{2}$}

\author{
${ }^{1}$ Department of Mathematics, Faculty of Education, Necmettin Erbakan University, Konya, Turkey. \\ ${ }^{2}$ Department of Mathematics, Faculty of Science, Karamanoglu Mehmetbey University, Karaman, Turkey.
}

Accepted 14 January, 2013

\begin{abstract}
There has been a great interest in studying difference equations and systems. One of the reasons for this is a necessity for some techniques which can be used in investigating equations arising in mathematical models describing real life situations in population biology, geometry, economics, probability theory, genetics, physics etc. In this paper, we investigate the solutions of the system of rational difference equations $x_{n+1}=\frac{x_{n-1}}{y_{n} x_{n-1}-1}, y_{n+1}=\frac{y_{n-1}}{x_{n} y_{n-1}-1}, z_{n+1}=\frac{x_{n} z_{n-1}}{y_{n}}$ where the initial values $x_{-1}, x_{0}, z_{-1}, z_{0}$ are real numbers and the initial values $y_{-1}, y_{0}$ are non-zero real numbers such that $x_{0} y_{-1}$ and $y_{0} x_{-1}$ are not equal to 1 . We give general solutions of the system. Also, we obtain necessary conditions for every solution of the system to be limited or unlimited.
\end{abstract}

Key words: Difference equation, system of difference equations, solutions.

\section{INTRODUCTION}

In this study, we investigate the behavior of the solutions of the difference equation system

$x_{n+1}=\frac{x_{n-1}}{y_{n} x_{n-1}-1}, y_{n+1}=\frac{y_{n-1}}{x_{n} y_{n-1}-1}, z_{n+1}=\frac{x_{n} z_{n-1}}{y_{n}}$

Where $x_{-1}, x_{0}, y_{-1}, y_{0}, z_{-1}, z_{0}$ are real numbers such that $y_{0} x_{-1} \neq 1, x_{0} y_{-1} \neq 1, y_{-1} \neq 0$ and $y_{0} \neq 0$.

Similar nonlinear systems of rational difference equations were investigated, for examples, Kurbanlı et al. (2011a) studied the behavior of the positive solutions of the system:

${ }^{*}$ Corresponding author. E-mail: akurbanli@yahoo.com.

$$
x_{n+1}=\frac{x_{n-1}}{y_{n} x_{n-1}+1}, y_{n+1}=\frac{y_{n-1}}{x_{n} y_{n-1}+1}
$$

Cinar (2004) studied the solutions of the system:

$$
x_{n+1}=\frac{1}{y_{n}}, y_{n+1}=\frac{y_{n}}{x_{n-1} y_{n-1}} .
$$

Kurbanli (2011b) studied the behavior of the solutions of the system:

$$
x_{n+1}=\frac{x_{n-1}}{y_{n} x_{n-1}-1}, y_{n+1}=\frac{y_{n-1}}{x_{n} y_{n-1}-1}, z_{n+1}=\frac{z_{n-1}}{y_{n} z_{n-1}-1} .
$$

Clark and Kulenović (2002) and Clark et al. (2003) 
investigated the global asymptotic stability of the system:

$$
x_{n+1}=\frac{x_{n}}{a+c y_{n}}, y_{n+1}=\frac{y_{n}}{b+d x_{n}} \text {. }
$$

Kulenović and Nurkanović (2005) studied the global asymptotic behavior of the solutions of the system:

$$
x_{n+1}=\frac{a+x_{n}}{b+y_{n}}, y_{n+1}=\frac{c+y_{n}}{d+z_{n}}, z_{n+1}=\frac{e+z_{n}}{f+x_{n}} .
$$

Zhang et al. (2006) investigated the behavior of the positive solutions of the system of difference equations:

$$
x_{n+1}=A+\frac{1}{y_{n-p}}, y_{n+1}=A+\frac{y_{n-1}}{x_{n-r} y_{n-s}} .
$$

Zhang et al. (2007) studied the boundedness, the persistence and global asymptotic stability of the positive solutions of the system:

$$
x_{n+1}=A+\frac{y_{n-m}}{x_{n}}, y_{n+1}=A+\frac{x_{n-m}}{y_{n}} .
$$

Yalcinkaya (2008) studied the global asmptotic stability of the system:

$$
z_{n+1}=\frac{t_{n} z_{n-1}+a}{t_{n}+z_{n-1}}, t_{n+1}=\frac{z_{n} t_{n-1}+a}{z_{n}+t_{n-1}} .
$$

\section{MAIN RESULTS}

\section{Theorem 1}

Let $\quad y_{0}=a, y_{-1}=b, x_{0}=c, x_{-1}=d, z_{0}=e, \mathrm{z}_{-1}=f$ be real numbers such that $a d \neq 1, \mathrm{cb} \neq 1, a \neq 0, b \neq 0$ and let $\left(x_{n}, y_{n}, z_{n}\right)$ be a solution of the system of Equation 1. Then all solutions of of Equation 1 are:

$$
\begin{aligned}
& x_{n}= \begin{cases}\frac{d}{(a d-1)^{\frac{n+1}{2}}}, & n \text {-odd } \\
c(c b-1)^{\frac{n}{2}}, & n \text {-even }\end{cases} \\
& y_{n}= \begin{cases}\frac{b}{(c b-1)^{\frac{n+1}{2}}}, & n-\text { odd } \\
a(a d-1)^{\frac{n}{2}}, & n-\text { even }\end{cases}
\end{aligned}
$$

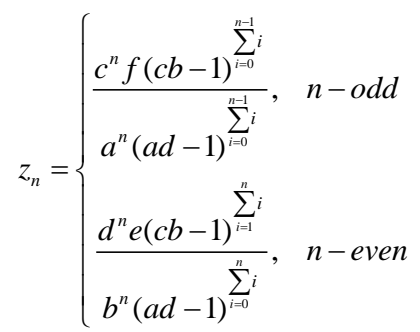

\section{Proof}

From Equation 1, we have

$$
\begin{aligned}
& x_{1}=\frac{x_{-1}}{y_{0} x_{-1}-1}=\frac{d}{a d-1}, \\
& y_{1}=\frac{y_{-1}}{x_{0} y_{-1}-1}=\frac{b}{c b-1}, \\
& z_{1}=\frac{x_{0} z_{-1}}{y_{0}}=\frac{c f}{a}, \\
& x_{2}=\frac{x_{01}}{y_{1} x_{0}-1}=\frac{c}{\frac{b}{c b-1} c-1}=c(c b-1),
\end{aligned}
$$$$
y_{2}=\frac{y_{0}}{x_{1} y_{0}-1}=\frac{a}{\frac{d}{a d-1} a-1}=a(a d-1),
$$$$
z_{2}=\frac{x_{1} z_{0}}{y_{1}}=\frac{\frac{d}{a d-1} e}{\frac{b}{c b-1}}=\frac{d e(c b-1)}{b(a d-1)},
$$

$x_{3}=\frac{x_{1}}{y_{2} x_{1}-1}=\frac{\frac{d}{a d-1}}{a(a d-1) \frac{d}{a d-1}-1}=\frac{d}{(a d-1)^{2}}$,

$$
y_{3}=\frac{y_{1}}{x_{2} y_{1}-1}=\frac{\frac{b}{c b-1}}{c(c b-1) \frac{b}{c b-1}-1}=\frac{b}{(c b-1)^{2}},
$$

$$
z_{3}=\frac{x_{2} z_{1}}{y_{2}}=\frac{c(c b-1) \frac{c f}{a}}{a(a d-1)}=\frac{c^{2} f(c b-1)}{a^{2}(a d-1)} .
$$

So Equations 2, 3 and 4 are true for $n=1,2,3$. Assume that Equations 2, 3 and 4 are true for $n=4,5, \ldots, k$. Then 


$$
\begin{aligned}
& x_{2 k-1}=\frac{x_{2 k-3}}{y_{2 k-2} x_{2 k-3}-1}=\frac{d}{(a d-1)^{k}}, \\
& x_{2 k}=\frac{x_{2 k-2}}{y_{2 k-1} x_{2 k-2}-1}=c(c b-1)^{k}, \\
& y_{2 k-1}=\frac{y_{2 k-3}}{x_{2 k-2} y_{2 k-3}-1}=\frac{b}{(c b-1)^{k}}, \\
& y_{2 k}=\frac{y_{2 k-2}}{x_{2 k-1} y_{2 k-2}-1}=a(a d-1)^{k}
\end{aligned}
$$

and

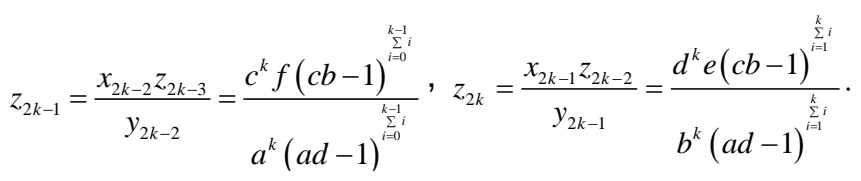

Now, we must show that Equations 2, 3 and 4 are true for $n=k+1$. From Equation 1, we have

$$
\begin{gathered}
x_{2 k+1}=\frac{x_{2 k-1}}{y_{2 k} x_{2 k-1}-1}=\frac{\frac{d}{(a d-1)^{k}}}{a(a d-1)^{k} \frac{d}{(a d-1)^{k}}-1}=\frac{d}{(a d-1)^{k+1}}, \\
y_{2 k+1}=\frac{y_{2 k-1}}{x_{2 k} y_{2 k-1}-1}=\frac{\frac{b}{(c b-1)^{k}}}{c(c b-1)^{k} \frac{b}{(c b-1)^{k}}-1}=\frac{b}{(c b-1)^{k+1}}
\end{gathered}
$$

and

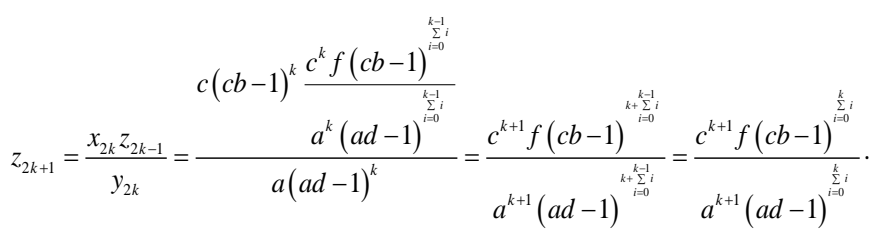

Also, we have

$$
\begin{aligned}
& x_{2 k+2}=\frac{x_{2 k}}{y_{2 k+1} x_{2 k-1}}=\frac{c(c b-1)^{k}}{\frac{b}{(c b-1)^{k+1}} c(c b-1)^{k}-1}=\frac{c(c b-1)^{k}}{\frac{b}{c b-1} c-1}=c(c b-1)^{k+1} \\
& y_{2 k+2}=\frac{y_{2 k}}{x_{2 k+1} y_{2 k}-1}=\frac{a(a d-1)^{k}}{\frac{d}{(a d-1)^{k+1}} a(a d-1)^{k}-1}=\frac{a(a d-1)^{k}}{\frac{d}{a d-1} a-1}=a(a d-1)^{k+1}
\end{aligned}
$$

and

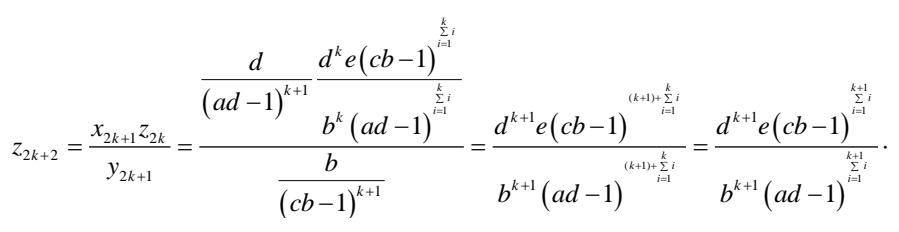

Therefore, the proof is completed by induction.

\section{Corollary 1}

Let $\left(x_{n}, y_{n}, z_{n}\right)$ be a solution of the system of Equation 1 and let $a, b, c, d$ be real numbers such that $a d \neq 1, c b \neq 1, a \neq 0$ and $b \neq 0$, then the following results hold:

(1) If $0<a, b, c, d, e, f<1$, then $\lim _{n \rightarrow \infty} x_{2 n-1}=\lim _{n \rightarrow \infty} y_{2 n-1}=\infty$ and $\lim _{n \rightarrow \infty} x_{2 n}=\lim _{n \rightarrow \infty} y_{2 n}=0$,

(2) If $0<a, b, c, d, e, f<1, \quad c<a$ and $c b=a d$ or $0<a, b, c, d, e, f<1, \quad c=a \quad$ and $\quad b<d, \quad$ then $\lim _{n \rightarrow \infty} z_{2 n-1}=0$,

(3) If $0<a, b, c, d, e, f<1, \quad c>a$ and $c b=a d$ or $0<a, b, c, d, e, f<1, \quad c=a \quad$ and $\quad d>b$, then $\lim _{n \rightarrow \infty} z_{2 n-1}=\infty$,

(4) If $0<a, b, c, d, e, f<1, \quad c=a$ and $b=d$, then $\lim _{n \rightarrow \infty} z_{2 n-1}=f$,

(5) If $0<a, b, c, d, e, f<1, d<b$ and $c b>a d$ or $0<a, b, c, d, e, f<1, d<b$ and $c b=a d$

or $0<a, b, c, d, e, f<1, f<1, b=d$ and $c>a$, then $\lim _{n \rightarrow \infty} z_{2 n}=0$,

(6) If $0<a, b, c, d, e, f<1, \quad d>b$ and $c b=a d$ or $0<a, b, c, d, e, f<1, \quad d=b$ and $a d<c b, \quad$ then $\lim _{n \rightarrow \infty} z_{2 n}=\infty$,

(7) If $0<a, b, c, d, e, f<1, d=b$ and $c=a$, then $\lim _{n \rightarrow \infty} z_{2 n}=e$.

\section{Proof}

(1) From $0<a, b, c, d<1$, we have $-1<a d-1<0$ and $-1<c b-1<0$. Hence, we obtain

$\lim _{n \rightarrow \infty} x_{2 n-1}=\lim _{n \rightarrow \infty} \frac{d}{(a d-1)^{n}}=d \lim _{n \rightarrow \infty} \frac{1}{(a d-1)^{n}}=\left\{\begin{array}{ll}-\infty, & n-\text { odd } \\ +\infty, & n-\text { even }\end{array}\right.$,

$\lim _{n \rightarrow \infty} y_{2 n-1}=\lim _{n \rightarrow \infty} \frac{b}{(c b-1)^{n}}=b \lim _{n \rightarrow \infty} \frac{1}{(c b-1)^{n}}= \begin{cases}-\infty, & n-\text { odd } \\ +\infty, & n-\text { even }\end{cases}$

and

$\lim _{n \rightarrow \infty} x_{2 n}=\lim _{n \rightarrow \infty} c(c b-1)^{n}=c \lim _{n \rightarrow \infty}(c b-1)^{n}=0$ 
$\lim _{n \rightarrow \infty} y_{2 n}=\lim _{n \rightarrow \infty} c(a d-1)^{n}=a \lim _{n \rightarrow \infty}(a d-1)^{n}=0$.

(2) From $0<a, b, c, d<1, c<a$ and $c b=a d$, we have $\frac{c b-1}{a d-1}=1$. Hence, we obtain

$\lim _{n \rightarrow \infty} z_{2 n-1}=\lim _{n \rightarrow \infty} \frac{c^{n} f(c b-1)^{\sum_{i=0}^{n-1} i}}{a^{n}(a d-1)^{\sum_{i=0}^{n-1} i}}=\lim _{n \rightarrow \infty} f\left(\frac{c}{a}\right)^{n}\left(\frac{c b-1}{a d-1}\right)^{\sum_{i=0}^{n-1} i}=0$.

Similarly, from $0<a, b, c, d<1, c=a$ and $b<d$, we have $\frac{c b-1}{a d-1}<1$. Hence, we obtain

$\lim _{n \rightarrow \infty} z_{2 n-1}=\lim _{n \rightarrow \infty} \frac{c^{n} f(c b-1)^{\sum_{i=0}^{n-1} i}}{a^{n}(a d-1)^{\sum_{i=0}^{n-1} i}}=\lim _{n \rightarrow \infty} f\left(\frac{c}{a}\right)^{n}\left(\frac{c b-1}{a d-1}\right)^{\sum_{i=0}^{n-1} i}=0$.

(3) From $0<a, b, c, d<1, c>a$ and $c b=a d$, we have $\frac{c b-1}{a d-1}=1$. Hence, we obtain

$\lim _{n \rightarrow \infty} z_{2 n-1}=\lim _{n \rightarrow \infty} \frac{c^{n} f(c b-1)^{\sum_{i=0}^{n-1} i}}{a^{n}(a d-1)^{\sum_{i=0}^{n-1} i}}=\lim _{n \rightarrow \infty} f\left(\frac{c}{a}\right)^{n}\left(\frac{c b-1}{a d-1}\right)^{\sum_{i=0}^{n-1} i}=\infty$.

Similarly, from $0<a, b, c, d<1, c=a$ and $d<b$, we have $\frac{c b-1}{a d-1}>1$. Hence, we obtain

$\lim _{n \rightarrow \infty} z_{2 n-1}=\lim _{n \rightarrow \infty} \frac{c^{n} f(c b-1)^{\sum_{i=0}^{n-1} i}}{a^{n}(a d-1)^{\sum_{i=0}^{n-1} i}}=\lim _{n \rightarrow \infty} f\left(\frac{c}{a}\right)^{n}\left(\frac{c b-1}{a d-1}\right)^{\sum_{i=0}^{n-1} i}=\infty$.

(4) From $0<a, b, c, d<1, c=a$ and $b=d$, we have $\frac{c b-1}{a d-1}=1$. Hence, we obtain

$\lim _{n \rightarrow \infty} z_{2 n-1}=\lim _{n \rightarrow \infty} \frac{c^{n} f(c b-1)^{\sum_{i=0}^{n-1} i}}{a^{n}(a d-1)^{\sum_{i=0}^{n-1} i}}=\lim _{n \rightarrow \infty} f\left(\frac{c}{a}\right)^{n}\left(\frac{c b-1}{a d-1}\right)^{\sum_{i=0}^{n-1} i}=f$.

(5) From $0<a, b, c, d<1, d<b$ and $b c>d a$, we have $\frac{c b-1}{a d-1}<1$. Hence, we obtain $\lim _{n \rightarrow \infty} z_{2 n}=\lim _{n \rightarrow \infty} \frac{d^{n} e(c b-1)^{\sum_{i=1}^{n} i}}{b^{n}(a d-1)^{\sum_{i=1}^{n} i}}=\lim _{n \rightarrow \infty} e\left(\frac{d}{b}\right)^{n}\left(\frac{c b-1}{a d-1}\right)^{\sum_{i=1}^{n} i}=0$.

Similarly, from $0<a, b, c, d<1, d<b$ and $b c=d a$, we have $\frac{c b-1}{a d-1}=1$. Hence, we obtain

$\lim _{n \rightarrow \infty} z_{2 n}=\lim _{n \rightarrow \infty} \frac{d^{n} e(c b-1)^{\sum_{i=1}^{n} i}}{b^{n}(a d-1)^{\sum_{i=1}^{n} i}}=\lim _{n \rightarrow \infty} e\left(\frac{d}{b}\right)^{n}\left(\frac{c b-1}{a d-1}\right)^{\sum_{i=1}^{n} i}=0$.

Similarly, from $0<a, b, c, d<1, d=b$ and $c>a$, we have $\frac{c b-1}{a d-1}<1$. Hence, we obtain

$\lim _{n \rightarrow \infty} z_{2 n}=\lim _{n \rightarrow \infty} \frac{d^{n} e(c b-1)^{\sum_{i=1}^{n} i}}{b^{n}(a d-1)^{\sum_{i=1}^{n} i}}=\lim _{n \rightarrow \infty} e\left(\frac{d}{b}\right)^{n}\left(\frac{c b-1}{a d-1}\right)^{\sum_{i=1}^{n} i}=0$.

(6) From $0<a, b, c, d<1, d>b$ and $b c=d a$, we have $\frac{c b-1}{a d-1}=1$. Hence, we obtain

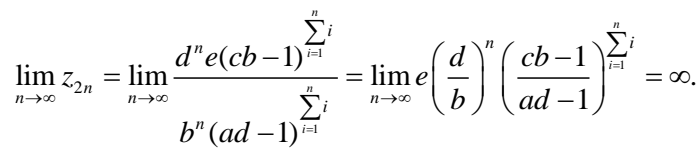

Similarly, from $0<a, b, c, d<1, d=b$ and $b c>d a$, we have $\frac{c b-1}{a d-1}>1$. Hence, we obtain

$\lim _{n \rightarrow \infty} z_{2 n}=\lim _{n \rightarrow \infty} \frac{d^{n} e(c b-1)^{\sum_{i=1}^{n} i}}{b^{n}(a d-1)^{\sum_{i=1}^{n} i}}=\lim _{n \rightarrow \infty} e\left(\frac{d}{b}\right)^{n}\left(\frac{c b-1}{a d-1}\right)^{\sum_{i=1}^{n} i}=\infty$.

(7) From $0<a, b, c, d<1, d=b$ and $c=a$, we have $\frac{c b-1}{a d-1}=1$. Hence, we obtain

$\lim _{n \rightarrow \infty} z_{2 n}=\lim _{n \rightarrow \infty} \frac{d^{n} e(c b-1)^{\sum_{i=1}^{n} i}}{b^{n}(a d-1)^{\sum_{i=1}^{n} i}}=\lim _{n \rightarrow \infty} e\left(\frac{d}{b}\right)^{n}\left(\frac{c b-1}{a d-1}\right)^{\sum_{i=1}^{n} i}=e$.

\section{Corollary 2}

Let $\left(x_{n}, y_{n}, z_{n}\right)$ be a solution of the system of Equation 1 
and let $a, b, c, d \in(1,+\infty)$ and $a>d>c>b$. If and

$a d-1, c b-1 \in(1,+\infty)$, then

$\lim _{n \rightarrow \infty} x_{2 n}=\lim _{n \rightarrow \infty} y_{2 n}=0$.

$\lim _{n \rightarrow \infty} x_{2 n-1}=\lim _{n \rightarrow \infty} y_{2 n-1}=\lim _{n \rightarrow \infty} z_{2 n-1}=0$,

and

$\lim _{n \rightarrow \infty} x_{2 n}=\lim _{n \rightarrow \infty} y_{2 n}=\infty$

\section{Proof}

From $\quad a, b, c, d \in(1,+\infty), \quad a>d>c>b \quad$ and

\section{Proof}

From $\quad a, b, c, d \in(1,+\infty), \quad a=c, b>d \quad$ and $a d-1, c b-1 \in(0,1)$, we have $\frac{c b-1}{a d-1}>1$. Hence, we have $\lim _{n \rightarrow \infty}(c b-1)^{n}=0$ and $\lim _{n \rightarrow \infty}(a d-1)^{n}=0$. Also, we have

$a d-1, c b-1 \in(1,+\infty)$, we have $\frac{c b-1}{a d-1}<1$. Hence, we have

$\lim _{n \rightarrow \infty} x_{2 n-1}=\lim _{n \rightarrow \infty} \frac{d}{(a d-1)^{n}}=d \cdot \lim _{n \rightarrow \infty} \frac{1}{(a d-1)^{n}}=+\infty$,

$\lim _{n \rightarrow \infty}(c b-1)^{n}=+\infty$ and $\lim _{n \rightarrow \infty}(a d-1)^{n}=+\infty$. Also, we have

$\lim _{n \rightarrow \infty} y_{2 n-1}=\lim _{n \rightarrow \infty} \frac{b}{(c b-1)^{n}}=b \cdot \lim _{n \rightarrow \infty} \frac{1}{(c b-1)^{n}}=+\infty$

$\lim _{n \rightarrow \infty} x_{2 n-1}=\lim _{n \rightarrow \infty} \frac{d}{(a d-1)^{n}}=d \cdot \lim _{n \rightarrow \infty} \frac{1}{(a d-1)^{n}}=0$,

and

$\lim _{n \rightarrow \infty} y_{2 n-1}=\lim _{n \rightarrow \infty} \frac{b}{(c b-1)^{n}}=b \cdot \lim _{n \rightarrow \infty} \frac{1}{(c b-1)^{n}}=0$

$\lim _{n \rightarrow \infty} z_{2 n-1}=\lim _{n \rightarrow \infty} \frac{c^{n} f(c b-1)^{\sum_{i=0}^{n-1} i}}{a^{n}(a d-1)^{\sum_{i=0} i} i}=\lim _{n \rightarrow \infty} f\left(\frac{c}{a}\right)^{n}\left(\frac{c b-1}{a d-1}\right)^{\sum_{i=0}^{n-1} i}=+\infty$

and

$\lim _{n \rightarrow \infty} z_{2 n-1}=\lim _{n \rightarrow \infty} \frac{c^{n} f(c b-1)^{\sum_{i=0}^{n-1} i}}{a^{n}(a d-1)^{\sum_{i=0} i} i}=\lim _{n \rightarrow \infty} f\left(\frac{c}{a}\right)^{n}\left(\frac{c b-1}{a d-1}\right)^{\sum_{i=0}^{n-1} i}=0$.

Similarly, we have

$\lim _{n \rightarrow \infty} x_{2 n}=\lim _{n \rightarrow \infty} c(c b-1)^{n}=c .0=0$

and

Similarly, we have

$\lim _{n \rightarrow \infty} y_{2 n}=\lim _{n \rightarrow \infty} a(a d-1)^{n}=a \cdot 0=0$.

$\lim _{n \rightarrow \infty} x_{2 n}=\lim _{n \rightarrow \infty} c(c b-1)^{n}=c \lim _{n \rightarrow \infty}(c b-1)^{n}=+\infty$

and

$\lim _{n \rightarrow \infty} y_{2 n}=\lim _{n \rightarrow \infty} a(a d-1)^{n}=a \lim _{n \rightarrow \infty}(a d-1)^{n}=+\infty$.

\section{REFERENCES}

Cinar C (2004). On the positive solutions of the difference equation system $x_{n+1}=\frac{1}{y_{n}}, y_{n+1}=\frac{y_{n}}{x_{n-1} y_{n-1}}$. Appl. Math. Comput. 158:303305.

Clark D, Kulenović MRS (2002). A coupled system of rational difference equations. Comput. Math. Appl. 43:849-867.

\section{Corollary 3}

Let $\left(x_{n}, y_{n}, z_{n}\right)$ be a solution of the system of Equation 1

Clark D, Kulenović MRS, Selgrade JF (2003). Global asymptotic behavior of a two-dimensional difference equation modelling competition. Nonlinear Anal. Teor. 52:1765-1776.

Kulenović MRS, Nurkanović Z (2005). Global behavior of a threedimensional linear fractional system of difference equations. J. Math. Anal. Appl. 310:673-689.

and let $a, b, c, d \in(1,+\infty)$ and $a=c, b>d$. If $a d-1, c b-1 \in(0,1)$, then

$\lim _{n \rightarrow \infty} x_{2 n-1}=\lim _{n \rightarrow \infty} y_{2 n-1}=\lim _{n \rightarrow \infty} z_{2 n-1}=\infty$,

Kurbanli AS (2011b). On the behavior of solutions of the system of rational difference equations $x_{n+1}=\frac{x_{n-1}}{y_{n} x_{n-1}-1}, y_{n+1}=\frac{y_{n-1}}{x_{n} y_{n-1}-1}, z_{n+1}=\frac{z_{n-1}}{y_{n} z_{n-1}-1}$. Discrete Dyn. Nat. Soc. 2011:1-12. 
Kurbanlı AS, Cinar C and Yalcinkaya I (2011a). On the behavior of positive solutions of the system of rational difference equations $x_{n+1}=\frac{x_{n-1}}{y_{n} x_{n-1}+1}, y_{n+1}=\frac{y_{n-1}}{x_{n} y_{n-1}+1}$. Math. Comput. Model. 53:12611267.

Yalcinkaya I (2008). On the global asymptotic stability of a second-order system of difference equations. Discrete Dyn. Nat. Soc. 2008:1-12.

Zhang Y, Yang X, Evans DJ, Zhu C (2007). On the nonlinear difference equation system $x_{n+1}=A+\frac{y_{n-m}}{x_{n}}, y_{n+1}=A+\frac{x_{n-m}}{y_{n}}$. Comput. Math.

Appl. 53:1561-1566.
Zhang Y, Yang X, Megson GM, Evans DJ (2006). On the system of rational difference equations $x_{n}=A+\frac{1}{y_{n-p}}, y_{n}=A+\frac{y_{n-1}}{x_{n-r} y_{n-s}}$. Appl. Math. Comput. 176:403-408. 